\title{
Interaction between Nonhistone Protein HMGB1 and Linker Histone H1 Facilitates the Formation of Structurally Ordered DNA-Protein Complexes
}

\author{
Alexander Polyanichko ${ }^{1,2}$ and Elena Chikhirzhina ${ }^{2}$ \\ ${ }^{1}$ Department of Molecular Biophysics, Faculty of Physics, Saint Petersburg State University, \\ Saint Petersburg 198504, Russia \\ ${ }^{2}$ Institute of Cytology, Russian Academy of Sciences, Saint Petersburg 194064, Russia \\ Correspondence should be addressed to Alexander Polyanichko, polyanichko@gmail.com \\ Copyright (C) 2012 A. Polyanichko and E. Chikhirzhina. This is an open access article distributed under the Creative Commons \\ Attribution License, which permits unrestricted use, distribution, and reproduction in any medium, provided the original work \\ is properly cited.
}

\begin{abstract}
The structural organization of the DNA complexes with nonhistone chromosomal protein and linker histone H1 was studied using circular dichroism spectroscopy (CD) and atomic force microscopy (AFM). It has been shown that due to the interaction between HMGB1 and H1 highly ordered DNA-protein complexes emerge in the solution. Their spectral properties are found to be similar to those of DNA/HMGB1-(AB) complexes, reported earlier. AFM images reveal the formation of fibrillike structures in the solution. We suggest that the electrostatic screening of the HMGB1 C-terminal domain by histone H1 facilitates stronger interaction of the HMGB1/H1 with DNA and the formation of the ordered supramolecular DNA-protein complexes.
\end{abstract}

Keywords: Chromatin, circular dichroism, DNA, HMGB1

\section{Introduction}

A great variety of DNA-binding proteins interact with DNA in cell nucleus-forming intricate DNAprotein complexes. There is a special class of "architectural" proteins in chromatin that perform structural functions. The family of HMGB-domain proteins is one of the best known examples of architectural proteins. The common feature of the proteins within the family is their DNA-binding motif, often called HMGB-domain [1, 2]. The structure of the HMGB-domain is highly conservative. Some of the proteins in this family contain only one HMGB domain, but a great number of the proteins possess two or more HMGB-domains. The members of the family are able to distinguish and preferably bind to distorted regions in DNA, such as four-way junctions, binding sites of the anticancer drug cisplatin, and great variety of other bends and crossovers [3]. Yet another abundant chromatin protein with somewhat similar properties is linker histone H1 [4-6], which binds to a linker DNA at the entrance/exit of the nucleosome. 
Functioning of these proteins in living cells requires the formation of large supramolecular complexes with DNA. When reconstructed in vitro such complexes very often tend to aggregate, which results in their precipitation. However, our previous studies revealed that at certain conditions the HMGB-domain proteins are able to induce the formation of the ordered multimolecular complexes upon binding to DNA [7, 8]. The aim of the present study is to investigate the structural organization of the HMGB1DNA complexes in presence of linker histone H1 using circular dichroism spectroscopy (CD) and atomic force microscopy (AFM).

\section{Materials and Methods}

To obtain the DNA-protein complexes, histone H1 (MW 21000) and nonhistone protein HMGB1 (MW 26500) were used. Both proteins were isolated from calf thymus as described earlier [9]. The protein content in the system was described in terms of the DNA base pairs to protein molar ratio $R$. For example, $R=70$ (or $70: 1$ b.p. to protein molecules ratio) means that there are 70 DNA base pairs for each protein molecule in the solution. In all complexes the H1 to HMGB1 molar ratio was maintained at $1: 1$. CD spectra of the complexes were recorded using CD6 dichrograph (Jobin-Yvon, France) in 5$\mathrm{mm}$ cylindrical quartz cells; the concentration of the DNA was maintained $0.06 \mathrm{mg} / \mathrm{mL}$. The complexes were prepared by the direct mixing of the protein and DNA solutions in the appropriate concentrations in presence of $15 \mathrm{mM} \mathrm{NaCl}$. The AFM images were obtained on mica in a tapping mode as described elsewhere [10].

\section{Results and Discussion}

Large DNA-protein complexes, when studied spectroscopically in UV region, very often cause considerable light scattering, which affects the shape of the spectral bands. In case of circular dichroism (CD) spectroscopy, it leads to the formation of characteristic spectral pattern, called $\psi$-type CD spectra (polymer and salt induced [11]). The appearance of such spectral pattern was attributed to electrostatic condensation of DNA in the presence of another polymer and at high concentration of counterions. However, sometimes instead of $\psi$-type CD spectra, it can be observed an increase in optical activity of DNA by orders of magnitude, attributed to the presence of large and highly ordered supramolecular DNA structures in the solution $[3,8,12-15]$. As we have shown earlier, the interaction of DNA with the HMGB-domain proteins results in some cases in the formation of structurally ordered supramolecular complexes, also inducing unusually strong optical activity of DNA [7, 8]. The formation of 30$\mathrm{nm}$ chromatin fibril is strongly dependent on the interaction of linker histone $\mathrm{H} 1$ and non-histone architectural proteins with DNA. To check whether the coaction of the histone H1 and non-histone protein HMGB1 is able to induce a spontaneous formation of ordered DNA-protein structures, we prepared artificial complexes between DNA and two chromosomal proteins: histone $\mathrm{H} 1$ and non-histone protein HMGB1. Both proteins are the most abundant chromatin proteins, and they play an important role in the structural organization of chromatin at the postnucleosomal level of its structural organization. These proteins are also able to initiate the formation of supramolecular structures when complexed with DNA, and each of them is able to induce $\psi$-type CD spectra of DNA, indicating strong intermolecular interaction present in the solution $[9,16]$. 


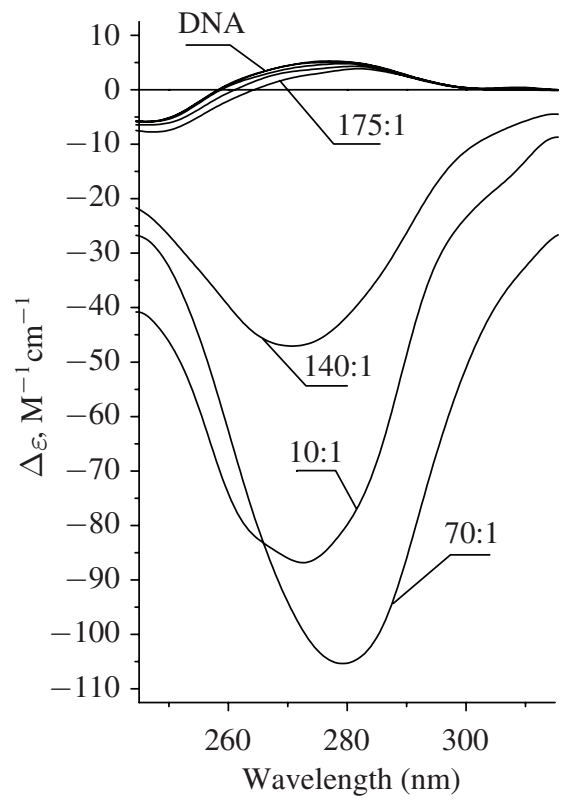

Figure 1: CD spectra of DNA and its complexes with non-histone protein HMGB1 and histone H1 at different DNA to protein ratios $R$, defined as the number of DNA base pairs per one protein molecule in the complex. The complexes with the ratios $175: 1 ; 140: 1 ; 70: 1 ; 10: 1$ are marked in the plot. Concentration of DNA in all samples was $0.06 \mathrm{mg} / \mathrm{mL}$; the optical path length was $0.5 \mathrm{~cm}$. The molar ratio of HMGB1 to $\mathrm{H} 1$ in the samples was maintained $1: 1$.

The typical UV-CD spectra of DNA and its complexes with the equimolar mixture of the proteins HMGB1 and H1 are given in Figure 1. At the DNA base pairs, to protein ratios above 175: 1 the spectra of the complexes reveal only minor gradual decrease of the intensity of the DNA band in vicinity of $275 \mathrm{~nm}$, indicating DNA-protein interactions in the major groove of the DNA double helix. Considerable light scattering of the samples with $R=140$ leads to the formation of the $\psi$-like CD spectra. Such $\mathrm{CD}$ spectra indicate that large multimolecular complexes are likely present in the solution. Further increase in protein content leads to increasing intensity of the DNA CD bands. Although at $R=70$ the light scattering is still considerable in the solution, the spectra are similar to those of DNA bound to the HMGB1-(AB) protein (Figure 2), which were attributed earlier to the highly ordered DNA-protein complexes [7]. The structural order of the DNA/HMGB1-(AB) complexes was extremely sensitive to the protein/DNA ratio and was observed in a very narrow $R$ interval. When the two proteins $\mathrm{H} 1$ and HMGB1are present in the system the CD spectra of DNA in the complex are sensitive, among the other factors, to the H1/HMGB1 ratio. In particular, using the above approach, we were able to obtain strong CD signal only in those complexes, in which H1 to HMGB1 molar ratio was close to 1.

To visualize the structures responsible for the strong optical activity of DNA in the complexes, we utilized atomic force microscopy. Using this approach the DNA-protein complexes with equimolar H1/HMGB1 ratio were studied. The typical images of the complexes with DNA base pairs to protein ratio $R=35$ are shown in Figure 3. The images reveal very extended fibril-like structures. Based on their linear dimensions, we conclude that each individual fibril is formed by several DNA molecules twisted 


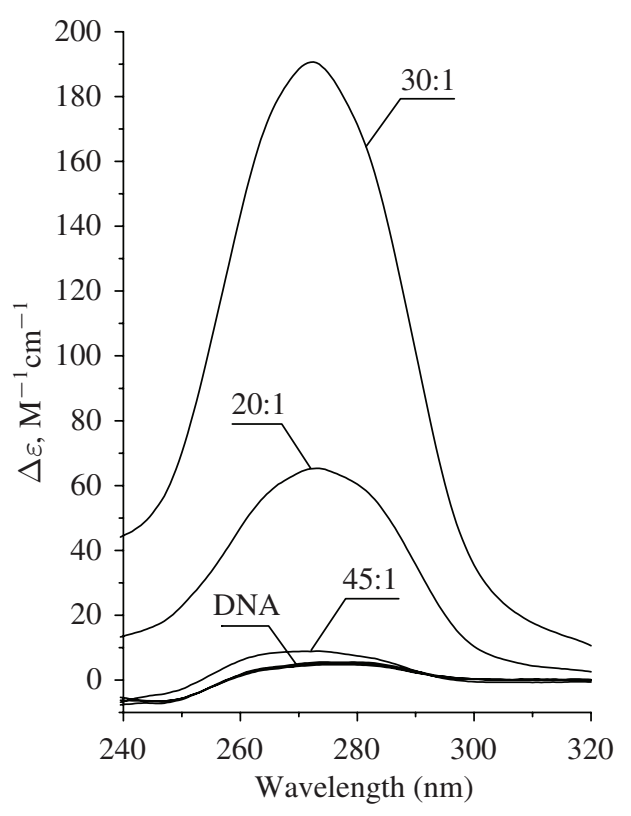

Figure 2: CD spectra of DNA and its complexes with recombinant protein HMGB1-(AB) at different DNA to protein ratios $R$, defined as the number of DNA base pairs per one protein molecule in the complex. The complexes with the ratios $45: 1 ; 30: 1 ; 20: 1$ are marked in the plot. Concentration of DNA in all samples was $0.03 \mathrm{mg} / \mathrm{mL}$; the optical path length was $1.0 \mathrm{~cm}$.
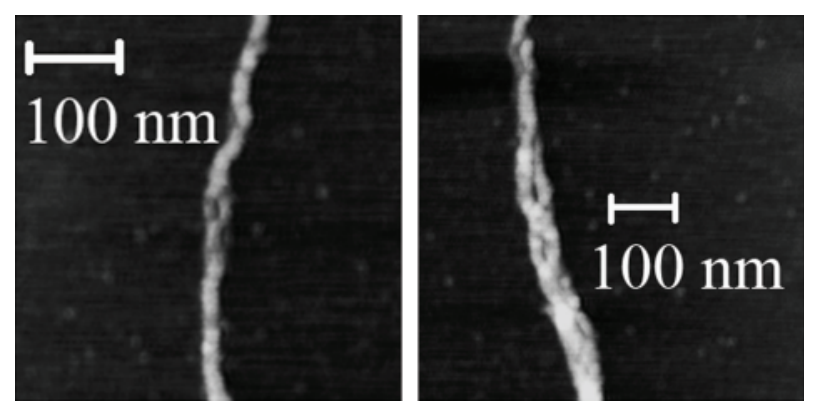

Figure 3: AFM height trace images of DNA-HMGB1/H1 complexes taken in a tapping mode on mica. DNA to protein ratios $R$, defined as the number of DNA base pairs per one protein molecule in the complex was $35: 1$. HMGB $1: \mathrm{H} 1$ molar ratio is $1: 1$. The bar scale corresponds to $100 \mathrm{~nm}$.

around each other. Individual DNA molecules within the fibril are held together by the protein molecules. The numerous DNA-protein and protein-protein interactions make the fibril rather stable. Most likely, the presence of the histone $\mathrm{H} 1$ leads to the partial neutralization of the negative charges of both the DNA phosphate groups and the C-terminal sequence of the HMGB1. The latter makes H1-HMGB1 complexes somewhat similar to the above HMGB1-(AB) protein, which lacks negatively charged Cterminal amino acid sequence but retains the HMGB domains available for binding DNA molecule. 
As we suggested earlier $[7,8]$ the neutralization of the negatively charged amino acid sequence of the HMGB1 is essential for the interaction of the both HMGB domains of the protein with DNA. Such a bidomain interaction facilitates, in turn, the formation of the highly ordered DNA-protein complexes. When the negatively charged tail of the HMGB1 is bound to the histone H1, it can have two major consequences: (i) both HMGB-domains of the HMGB1 are able to interact with DNA, forming stronger DNA-protein complexes; (ii) the HMGB1-H1 protein complexes are able to produce intermolecular DNA cross-links very effectively. Both of these factors facilitate the formation of supramolecular complexes, observed above.

Thus, the electrostatic screening of the HMGB1 C-terminal domain by histone $\mathrm{H} 1$ facilitates stronger interaction of the HMGB1/H1 with DNA and the formation of the ordered supramolecular complexes.

\section{Acknowledgments}

The authors are grateful to the Russian Foundation for Basic Researches (RFBR) (Grant 09-0801119/12-08-01134), the Government of St. Petersburg, Federal program, "Scientific and pedagogical labor force for an innovative Russia (GC P1163)" for their financial support. They are also grateful to Dr. Zoya Leonenko for her help with AFM experiments.

\section{References}

[1] C. M. Read, P. D. Cary, C. Crane-Robinson, P. C. Driscoll, and D. G. Norman, "Solution structure of a DNA-binding domain from HMG1," Nucleic Acids Research, vol. 21, no. 15, pp. 3427-3436, 1993.

[2] J. O. Thomas and A. A. Travers, "HMG1 and 2, and related 'architectural' DNA-binding proteins," Trends in Biochemical Sciences, vol. 26, no. 3, pp. 167-174, 2001.

[3] A. Travers, "Recognition of distorted DNA structures by HMG domains," Current Opinion in Structural Biology, vol. 10, no. 1, pp. 102-109, 2000.

[4] A. Travers, "The location of the linker histone on the nucleosome," Trends in Biochemical Sciences, vol. 24, no. 1, pp. 4-7, 1999.

[5] J. Widom, "Structure, dynamics, and function of chromatin in vitro," Annual Review of Biophysics and Biomolecular Structure, vol. 27, pp. 285-327, 1998.

[6] J. Zlatanova and J. Yaneva, "Histone H1-DNA interactions and their relation to chromatin structure and function.," DNA and Cell Biology, vol. 10, no. 4, pp. 239-248, 1991.

[7] E. Chikhirzhina, A. Polyanichko, Z. Leonenko, H. Wieser, and V. Vorob'Ev, "C-terminal domain of nonhistone protein HMGB1 as a modulator of HMGB1-DNA structural interactions," Spectroscopy, vol. 24, no. 3-4, pp. 361-366, 2010.

[8] A. M. Polyanichko, E. V. Chikhirzhina, A. N. Skvortsov et al., "The HMG1 Ta(i)le," Journal of Biomolecular Structure and Dynamics, vol. 19, no. 6, pp. 1053-1062, 2002.

[9] E. V. Chikhirzhina, A. M. Polyanichko, A. N. Skvortsov, E. I. Kostyleva, C. Houssier, and V. I. Vorob'ev, "HMG1 domains: the victims of the circumstances," Molecular Biology, vol. 36, no. 3, pp. 412-418, 2002.

[10] A. M. Polyanichko, Z. V. Leonenko, D. Cramb, H. Wieser, V. I. Vorob'ev, and E. V. Chikhirzhina, "Visualization of DNA complexes with HMGB1 and its C-truncated form HMGB1(A+B)," Biophysics, vol. 53, no. 3, pp. 202-206, 2008. 
[11] C. F. Jordan, L. S. Lerman, and J. H. Venable, "Structure and circular dichroism of DNA in concentrated polymer solutions.," Nature: New Biology, vol. 236, no. 64, pp. 67-70, 1972.

[12] V. A. Beliakov, V. P. Orlov, S. V. Semenov et al., "Circular dichroism spectral characteristics of liquid-CRYSTAL dispersions of double-stranded DNA and DNA complexes with dyes," Biofizika, vol. 41, no. 5, pp. 1044-1055, 1996.

[13] I. M. Evdokimov and I. M. Varshavskii, "Liquid-crystal state of nucleic acids in solution," Doklady Akademii nauk SSSR, vol. 263, no. 5, pp. 1254-1257, 1982.

[14] Y. M. Evdokimov, V. I. Salyanov, N. M. Akimenko, and Y. M. Varshavskii, "Compact form of DNA in solution. 10. Characteristics of circular-dichroism spectra of compact particles of DNA formed in water-salt solutions containing polyethylene-glycol," Molecular Biology, vol. 11, pp. 230-235, 1977.

[15] M. Evdokimov Yu. M. and V. I. Salyanov, "Characteristic features of circular dichroism of liquid crystals formed from DNA complexes with some antibiotics," Antibiotiki, vol. 28, no. 4, pp. 266270, 1983.

[16] A. Polyanichko and H. Wieser, "Fourier transform infrared/vibrational circular dichroism spectroscopy as an informative tool for the investigation of large supramolecular complexes of biological macromolecules," Biopolymers, vol. 78, no. 6, pp. 329-339, 2005. 


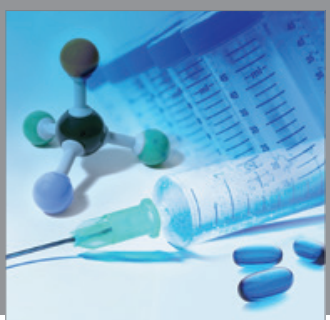

International Journal of

Medicinal Chemistry

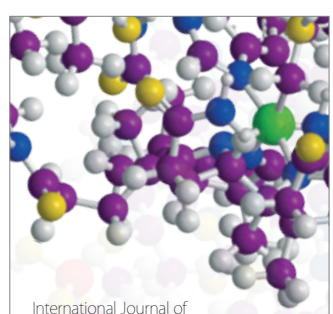

Carbohydrate Chemistry

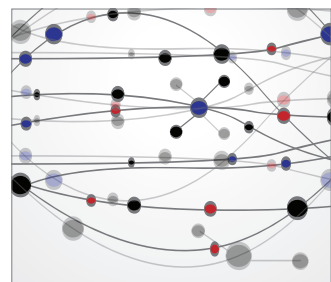

The Scientific World Journal
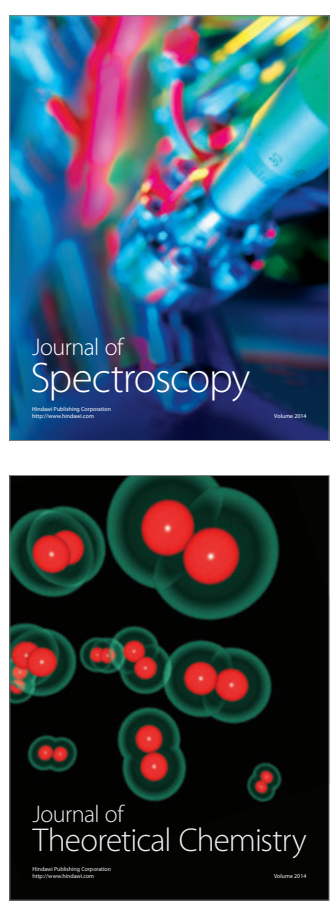
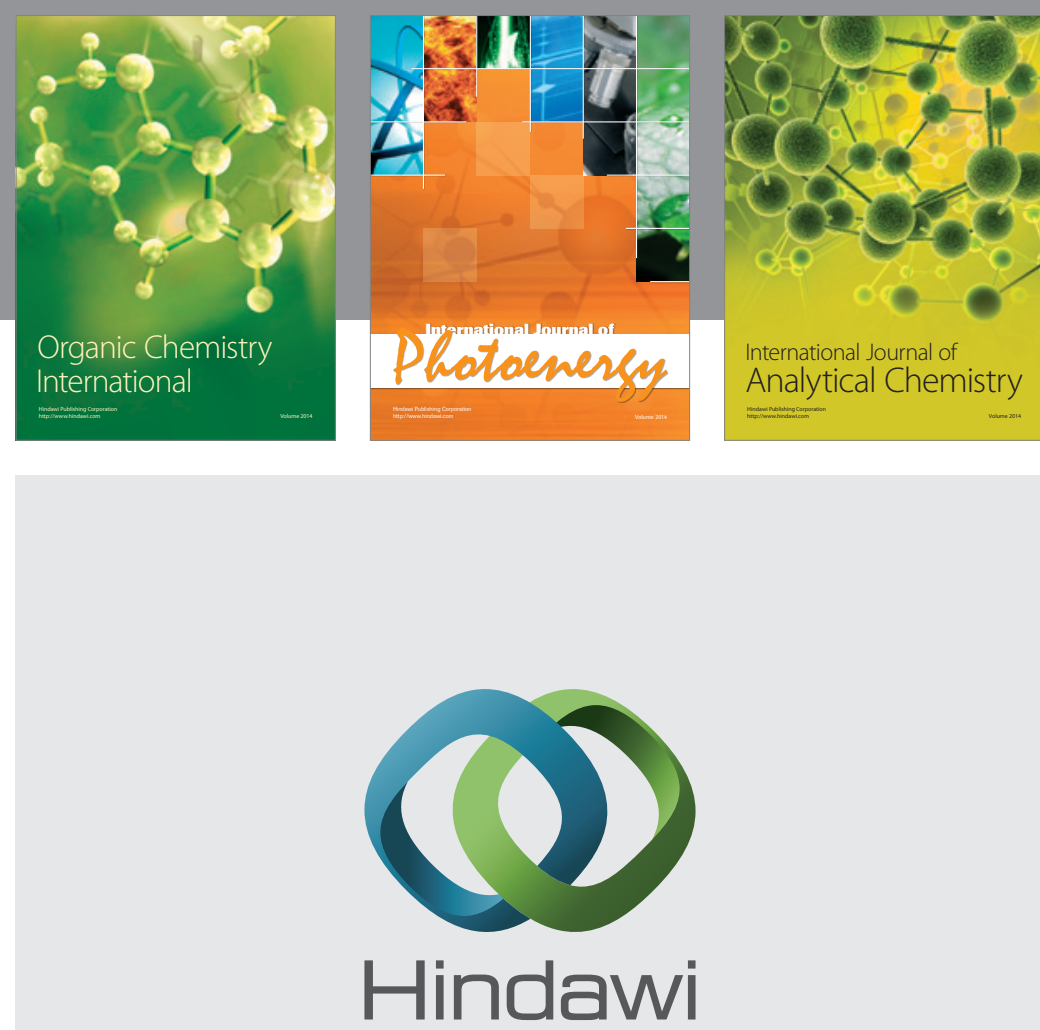

Submit your manuscripts at

http://www.hindawi.com
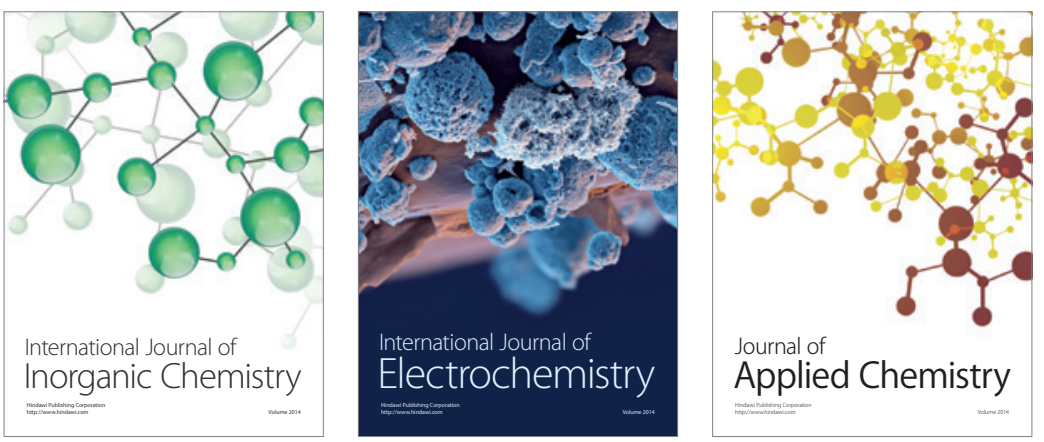

Journal of

Applied Chemistry
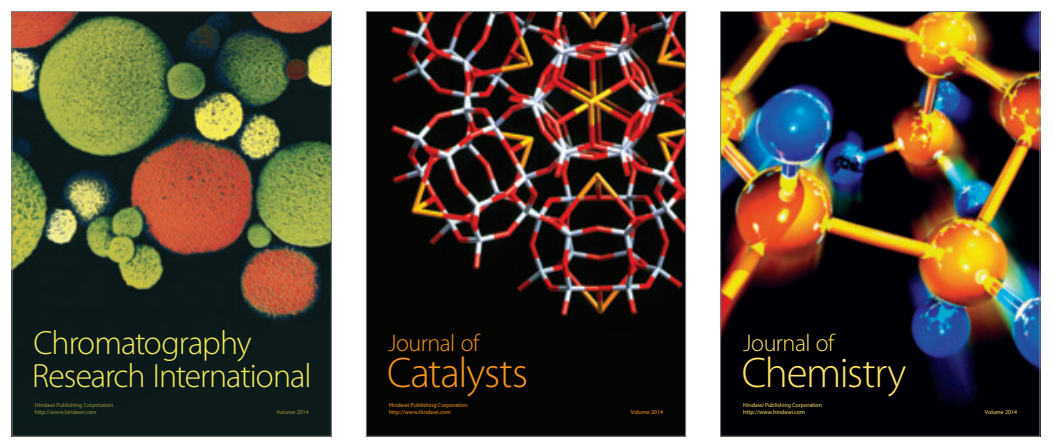
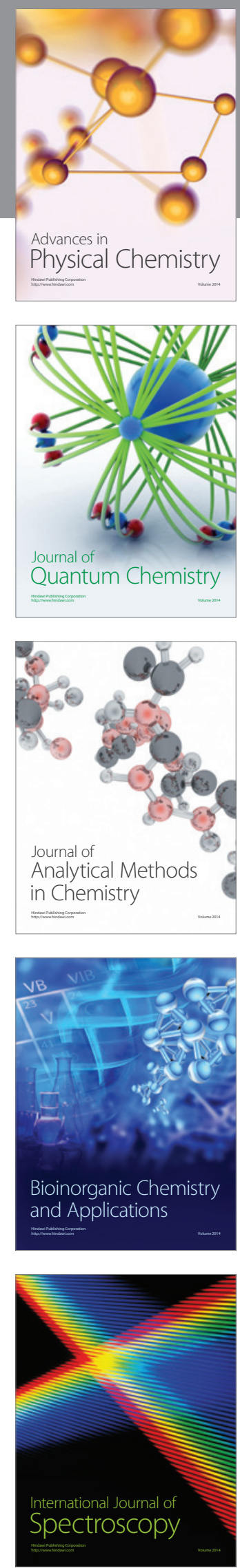